\title{
AUTOMATED DATA ENTRY SYSTEM FOR WEB-PORTAL USING ROBOTIC PROCESS AUTOMATION
}

\author{
Rajat, Pinki Nayak \\ E-Mail Id: ${ }^{1}$ rajat3014@gmail.com, ${ }^{2}$ pnayak@amity.edu \\ Department of Computer Science and Engineering, Amity School of Engineering and Technology, Noida, \\ India
}

\begin{abstract}
The main objective of Robotic Process Automation (RPA) is to improve client experience and effective excellence by improving efficiency, performance, and agility of the process performed. RPA technology consists of a software robot installed in a user's computer, machine or device that tracks repetitive human activities and replicate them to perform the complicated, redundant, and rule-based task at ease which is performed on a daily basis.

In data entry operations, which includes manual entry of data containing various fields, collected from multiple sources and combined together in organized form, and is needed to be uploaded on a particular website in a required manner could easily be automated using UI-Path which is a tool to implement Robotic Process Automation. After the task of data entry operation on required website is completed, an acknowledgement in the form of e-mail is sent to user that the task has been completed.
\end{abstract}

Keywords: RPA, UI-Path, Automation, Data Entry, Web Automation, Orchestrator.

\section{INTRODUCTION}

Management is the word used to organize. Being a good manager means that one is a very organized person and can handle things better than others. Automated order management using robot process automation is the future of the way sales are processed. RPA is a technology that mimics the human action that are associated with a multitude of businesses. In the order processing industry, RPA offers a lot of benefits by automating the order processing and fixing all the difficulties and loopholes of this industry with the help of various software. Automated order management using robot process automation saves times, handles complex workflow, flexible and gives high return investment. In manual process, data is gathered from multiple sources and is organized into a required format mainly into excel sheets. Then the process of data entry operations is performed by making an entry of each of the data from excel sheet to the desired web portal according to the format which generally consists of multiple attributes for each user.

Since all the task is done manually, a lot of time is required to complete the task and also there is a possibility of human error while making an entry from excel sheet to the desired web portal. Due to the problems mentioned above, the staff members performing data entry operations had to invest a lot of time in determining the instructions, comparing the data after uploading onto web portal to check for errors. The entire process when done manually for a data containing 5 attributes for 100 users usually takes around 1 hour 30 minutes, but when performed using a robot developed using RPA takes around 30 minutes thus reducing the amount of time by 1 hour which is surely an inordinate amount of time, to perform data entry of just 100 users.

In automated management of data, the management of the data which generally contains uploading the data on a particular server for further operations is the first and basic step to perform further operations using this data, thus data management is the most important factor that is needed in any business. Automated management of data is that the data is managed by preset robotic software which needs a onetime input, though regular updates are required to customize the robot according to user's demand, and the rest of the processing of data is done automatically. Thus, removing the need of any manpower, so that they can perform more creative work instead of performing a repetitive task and thus building a future that will be time saving and human friendly.

\section{RESEARCH QUESTIONS}

By keeping the law of the situation and the demand to know about what is going to happen, this research demarcates the following two research questions (RQ):

RQ1: How can a data entry task be automated with multiple fields and for multiple users?

RQ2: How is the robot deployed for automation better?

\section{LITERATURE REVIEW}

\subsection{Robotic Process Automation}

Robotic Process Automation is the technology which allows anybody today to design computer software, or a "robot" and so called a "software robot" to mimic and integrate the activities of a human interacting with digital systems to execute a business process. RPA robots utilize the user interface to obtain data and control applications just in the same way as humans do. They infer, trigger responses, and connect with other systems to perform on a vast variety to perform tedious tasks. Only substantially better: These RPA software robots never sleeps, makes zero mistakes, and costs a lot less than an employee. 


\subsection{Working of Robotic Process Automation}

RPA robots are capable of mirroring many-if not most-human user actions. They log into applications, move files and folders, copy, and paste data, fill in forms, obtain structured and semi-structured data from documents, scraping browsers, and many more. RPA or Robotic Process Automation is a technology that mimics the human actions that are associated with a multitude of businesses. The 'robots' involved in this process are not the robots in a physical sense, rather they are the software program that are used in computers and works with all business applications.

\subsection{UI-Path Tool for RPA}

UI path is one of the widely used robotic process automation tools and it was founded by the Romanian entrepreneur in the year 2015 to provide software which can eliminate the tedious time-consuming back office works. It converts boring tasks into automated ones with the help of unique tools. It enables your processes to be more effective and reliable. UI-Path is a global software company that develops a platform for Robotic Process Automation and the tool is used to automate the recurring and redundant tasks and removes human involvement. The tool is simple and user friendly which have a drag and drop functionality of activities. UI-path is an advanced tool that is used to design automated processes visually, through diagrams. UI-path robot executes the processes which are built in Studio part of tool, mimicking the human activity. Robots can work attended (require human input for functioning) or unattended (run without human supervision in any environment, be it virtual or not) or as assistants. In UI-Path, the Automation is associated with multiple aspects and it enables you to automate the rule-based process. To achieve effective automation, each work should be assigned to a specific thing and can be performed effectively. Each activity in UIPath must undergo small activities like reading a file, clicking a button, writing a log panel, etc.

These are some of the essential workflows of the UI-path:

\subsubsection{Sequence}

It best suits for the linear process in moving from one stage to another without causing any interruption to the workflow.

\subsubsection{Flowcharts}

It is designed to suit the organizations whose automation requires some logical ability. It clears all the problems in a diversified manner through multiple branching operations.

\subsubsection{State Machine}

It is well designed to suit the needs of the organizations which have huge workflows and a large number of transactions. State machine uses an infinite number of states in their execution which are triggered by a condition (transition) or task.

\subsection{Automated Management of Data}

RPA assists data entry operations to achieve a high degree of precision especially in areas like re-keying which are more susceptible to human errors. This is because RPA can automate some stages of the process thereby enabling humans to take considered and smart decisions in the course of manual data entry cycle. It will improve quality, reduce time, and save the data rework costs are the primary plus points of using it in the data entry operations.

\subsection{Benefits of RPA in Data Entry}

\subsubsection{Save Time}

The RPA robots work behind the scene, verify the data, pulled out data from multiple systems, customer related information within the time span of a few seconds. In addition to this, they also ensure that there aren't any duplicate orders. And if it is by any chance, the order is further sent to a staff member who resolves the issue manually. This means the entire task which previously took around 20 minutes to solve can now be done within seconds, and with more accuracy and precision.

\subsubsection{Handling Complex Workflows}

Earlier when the workload used to be complex or more than usual, the staff used to resort to 'swivel-seating' process between multiple systems wherein the staff members would spend an exorbitant amount of time in completing data entry process in required format. Such slow service with somy typing or human errors could impact negatively. But with the right blend of RPA tools, the multi-system work can be effectively done in a nonintrusive manner due to which handling or accommodating complex workflows becomes easier than ever.

\subsubsection{Round the Clock Operational Feasibility}

Since RPA does not require any manual input, the software will make $24 * 7$ operations feasible. Also, the speed of performing tasks of software is many times faster than a human. This will decrease the cycle times and enhance the accuracy and throughput. Thus a large amount of task could be completed in the same time as compared to manual operation.

\subsubsection{Flexibility}

DOI Number: https://doi.org/10.30780/specialissue-ICACCG2020/027 
Flexibility is an add-on benefit of using RPA for automating the data entry process. Once you define the process and lay down the instructions that the robot software can execute, you can schedule the operation any time. Also, any number of robots can be deployed to work upon the project. In addition to this, you can always reassign tasks to a robot in case there is an influx of other important processes.

\subsubsection{High Returns on Investment}

RPA offers financial benefits to the company as well. Of course, such software can surely seem expensive but the cost of hiring additional employees to manage the data entry operations and the costly admin mistakes made due to a human error far surpass the cost of software. This is surely an attractive proposition for the data entry industry.

The operating cost of a robot is much lesser as compared to the cost of maintaining a full time labour force or employees and it also adds to the quality of service being delivered. There are many other additional benefits that RPA offers which also reinforce the fact that the software is and will continue to transform the future of data entry operations using automation.

\subsection{Orchestrator}

As UI-Path tool provides a server like feature known as orchestrator from where these software bots could be operated remotely from any device to accomplish the task for which the process is generated in UI-Path studio, even when UIPath is not installed on the device from where the robot is triggered to complete the task.

Orchestrator provides appropriate environment and the admin can assign an unattended or a floating robot as per the requirement to the process created for completing the task for which the robot is created.

\subsection{Acknowledgement of Data}

After the task of data entry operation onto the web portal is completed, acknowledgement for the same is received by triggering an email to the admin that the task assigned to the robot is completed successfully.

\section{RESEARCH METHODOLOGY}

This paper contains the process of automating a data entry task for various users and each user holding multiple fields of data to be supplied on a web portal. The task of automating is done by using Robotic Process Automation technique, and the tool used to implement it is UI-Path.

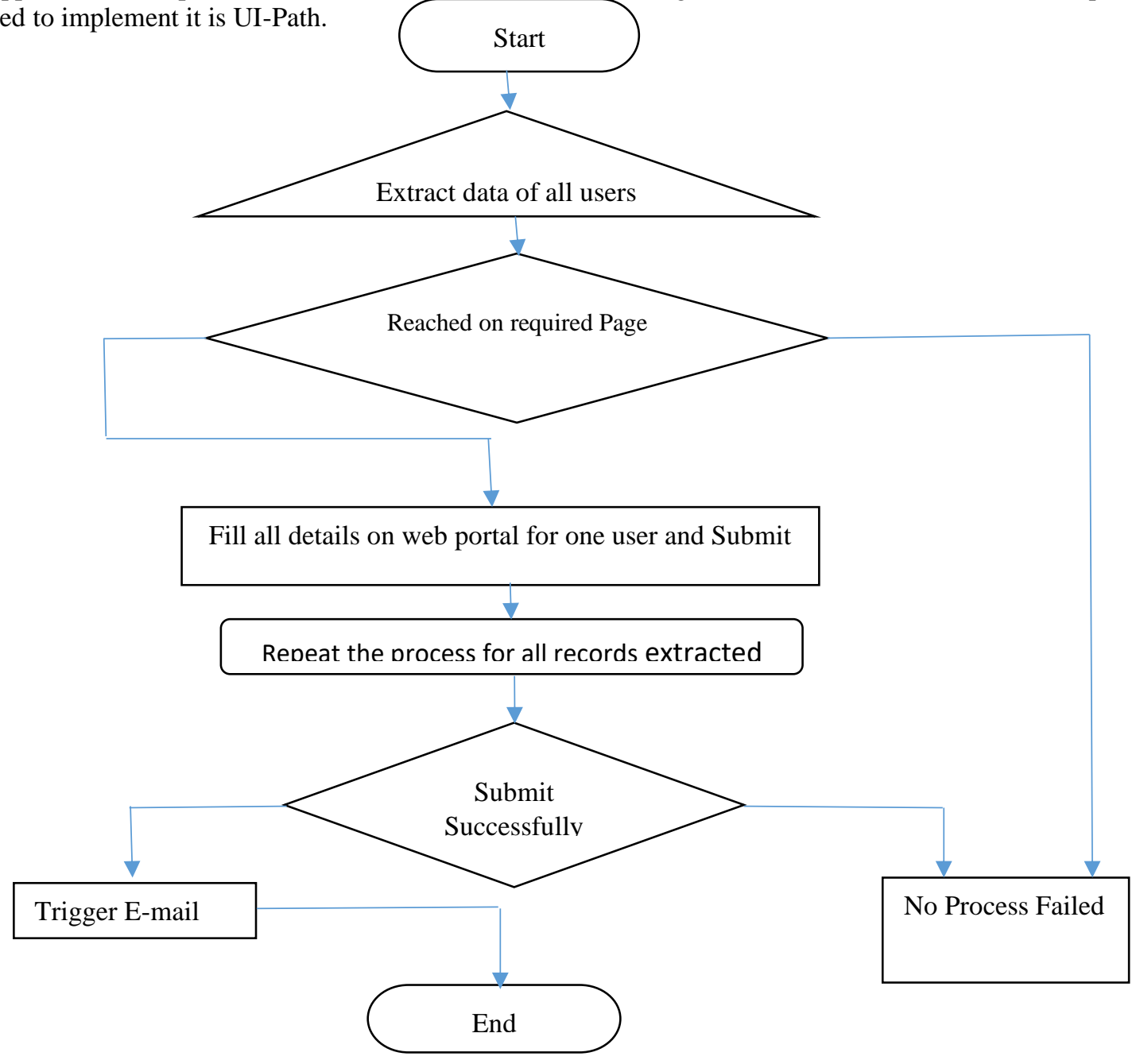

Fig. 4.1 Flowchart of Process for Automated Data Entry 
The data of all users is initially provided in an excel sheet, so the data is first extracted from the excel sheet to the UIPath tool, and it is then stored as an internal database for faster access. After extraction of complete data, the required web-portal, where data entry is to be done is reached by hitting the URL. On reaching the desired page, the bot starts the data entry operation for the $1^{\text {st }}$ user and fills in all the fields by accessing data from the internal database. After the data entry is complete for the $1^{\text {st }}$ user, the bot starts the data entry for $2^{\text {nd }}$ user and repeats the entire process for all the users. After the task is completed for all the users, the bot triggers an e-mail to the admin that the data entry task assigned to the robot is now complete, and the robot could now be deployed for another task. All this operation could be operated from virtual device by using the server like application known as orchestrator, which provides a runnable environment to the robot, even if UI-Path tool to implement Robotic Process Automation is not installed on the device. Fig. 4.1 shows the complete process flow for automated data entry operation in the form of a flowchart. The data is initially extracted from the source, and after reaching onto desired web portal, the robot starts to fill the entries of data extracted, thus completing the data entry process.

\section{ANALYSIS AND FINDINGS}

The approach is to extract data from multiple sources and arrange it in an organized form in an excel sheet for data entry process.

\subsection{Excel Extraction}

In Excel extraction, the complete data gathered from multiple sources and is present in organized form in excel sheet is extracted row by row by applying for each row process of excel extraction in UI-Path. After the data is extracted from excel sheet, it is stored as an internal database in UI-Path tool, for fast access of data and once the package of process is created, the data gets attached to the process.

\subsection{Web Automation}

Web Automation includes navigating to the web portal, where data entry task is to be accomplished. After navigating, the robot will check the fields that which data needs to be uploaded into a particular field, and after checking the robot will pull data present in excel sheet in the related field and will complete the data entry task. The process is repeated for multiple fields of a single user and the whole process is repeated for multiple users and the details are stored onto web portal.

Fig 5.1 shows the data of various users, containing multiple fields, filled by the robot on the desired web-portal

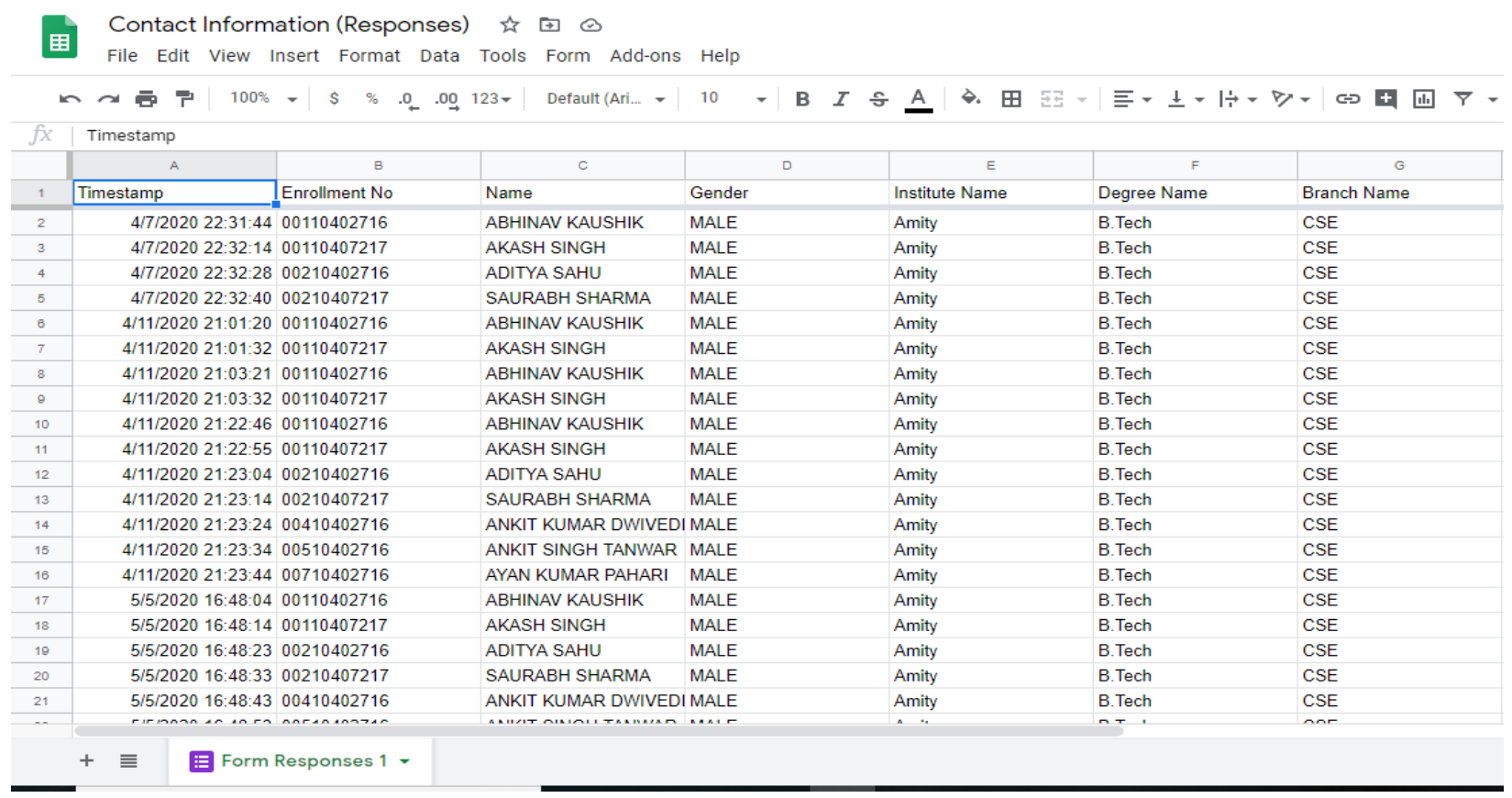

Fig. 5.1 Response Filled by Robot in Different Fields Present on Web Portal

\subsection{E-Mail Automation}

After the complete data from excel sheet is uploaded to the web portal and the data entry operation is completed, a confirmatory acknowledgement in the form of E-mail is triggered to the admin that the task is completed and the robot deployed is now free to perform another task.

For sending E-mail, Simple Mail Transfer Protocol (SMTP) is used and port no 587 is used which is meant for email client to email server communication-sending out email using SMTP protocol and TLS security encryption. The server used for sending email is "smtp.gmail.com" which is a google server to access Gmail and credentials for login to Gmail account are passed as parameters. 
Fig. 5.2 shows the final mail received to the admin that the task deployed to the robot is now completed successfully, so all the data entries are made on the desired web-portal and the robot is free and another task could now be deployed to the robot.

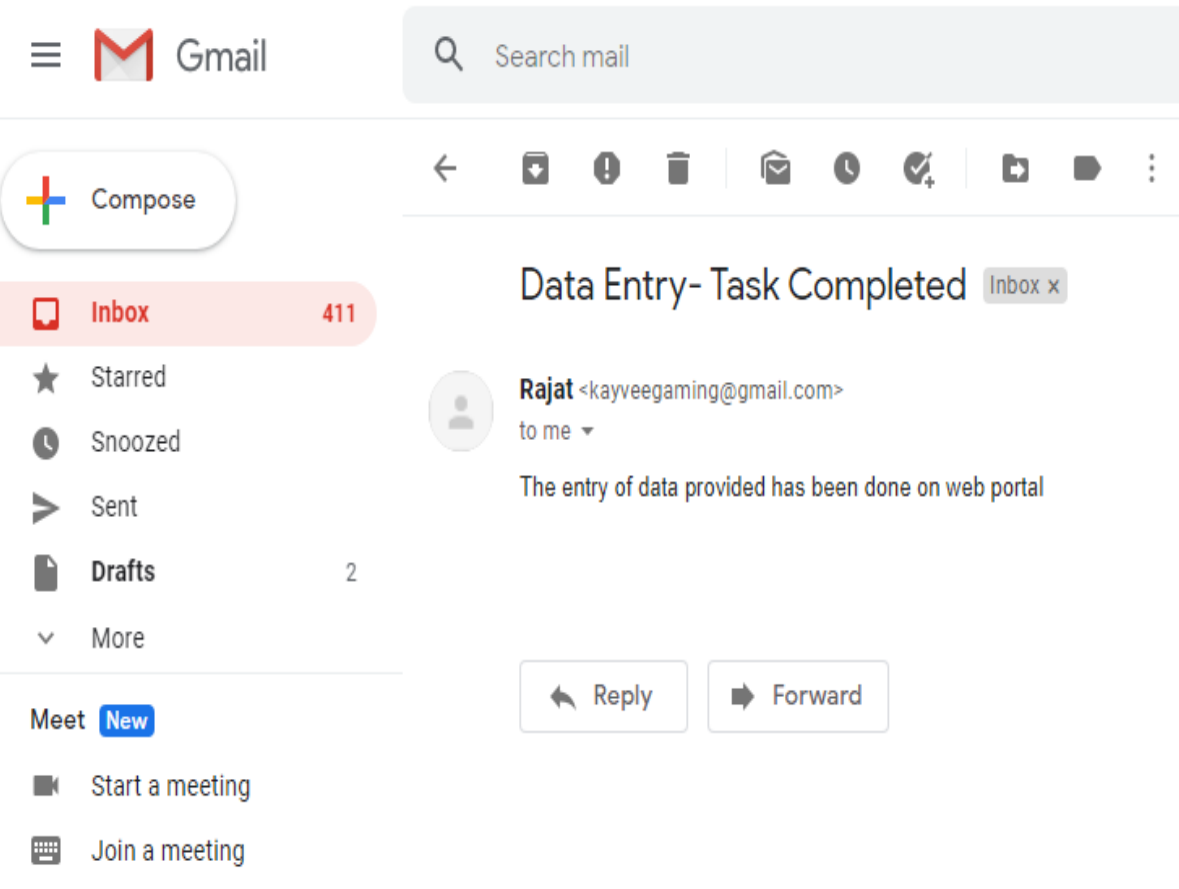

Fig. 5.2 Final Mail Received After Completion of Task

\section{DISCUSSION AND IMPLICATIONS}

In this study, we found that the data entry task which requires a lot of human effort, could be automated by implementing Robotic Process Automation, which reduces the time taken to complete the task by a large extent. Also if the task is completed manually, there is always a risk of human error, which is not present if robot is deployed for the process and the robot could work $24 \times 7$, which is ideally not possible for any human being. Thus by automating the data entry task by a robot, the process becomes more reliable, efficient, feasible and allows human beings to do much creative work instead of a repetitive task.

\section{REFERENCES}

[1] Lacity, M., Willcocks,LP., and Craig, A. (2015), "Robotic Process Automation at Telefónica O2," The Outsourcing Unit Working Research Paper Series

[2] Automation Of a Business Process Using Robotic Process Automation (rpa): A Case Study Santiago AguirreAlejandro Rodriguez - Communications in Computer and Information Science Applied Computer Sciences in Engineering - 2017

[3] https://academy.uipath.com/learn

[4] https://forum.uipath.com/ 